\title{
Other articles noted 14 Nov 2003 to 30 Jan 2004
}

A list of the journals that are reviewed and the criteria for selecting articles from these journals for inclusion in EvidenceBased Nursing are included in each issue. The following articles passed all criteria but were not abstracted because, in the judgment of the editors, their findings were less widely applicable to nursing practice, the topic was of interest to only a select group of nurse specialists, or the topic was recently addressed in another abstract.

\section{TREATMENT}

Abramson MJ, Puy RM, Weiner JM. Allergen immunotherapy for asthma. Cochrane Database Syst Rev 2003;(4):CD001186. http://www.ncbi.nlm.nih.gov/entrez/query.fcgi?cmd $=$ Retrieve $\& \mathrm{db}=$ PubMed\&list_uids = 14583928\&dopt $=$ Abstract

Airaksinen OV, Kyrklund N, Latvala K, et al. Efficacy of cold gel for soft tissue injuries: a prospective randomized doubleblinded trial. Am J Sports Med 2003;31:680-4.

http://www.ncbi.nlm.nih.gov/entrez/query.fcgi?cmd

$=$ Retrieve \&db $=$ PubMed\&list_uids $=$ 12975186\&dopt

$=$ Abstract

Alam NH, Hamadani JD, Dewan N, et al. Efficacy and safety of a modified oral rehydration solution (ReSoMaL) in the treatment of severely malnourished children with watery diarrhea. J Pediatr 2003;143:614-9.

http://www.ncbi.nlm.nih.gov/entrez/query.fcgi?cmd

$=$ Retrieve \&db $=$ PubMed\&list_uids = 14615732\&dopt

$=$ Abstract

Anderson AD, McNaught CE, MacFie J, et al. Randomized clinical trial of multimodal optimization and standard perioperative surgical care. Br J Surg 2003;90:1497-504. http://www.ncbi.nlm.nih.gov/entrez/query.fcgi?cmd $=$ Retrieve $\& \mathrm{db}=$ PubMed\&list_uids $=14648727 \& \mathrm{dopt}$ $=$ Abstract

Bacaltchuk J, Hay P. Antidepressants versus placebo for people with bulimia nervosa. Cochrane Database Syst Rev 2003; (4):CD003391.

http://www.ncbi.nlm.nih.gov/entrez/query.fcgi?cmd

$=$ Retrieve $\& \mathrm{db}=$ PubMed\&list_uids $=$ 14583971\&dopt

$=$ Abstract

Badamgarav E, Weingarten SR, Henning JM, et al. Effectiveness of disease management programs in depression: a systematic review. Am J Psychiatry 2003;160:2080-90. http://www.ncbi.nlm.nih.gov/entrez/query.fcgi?cmd

$=$ Retrieve \&db $=$ PubMed\&list_uids $=14638573 \& d o p t$

$=$ Abstract

Bedi A, Murray JM, Dingley J, et al. Use of xenon as a sedative for patients receiving critical care. Crit Care Med 2003;31:2470-7.

http://www.ncbi.nlm.nih.gov/entrez/query.fcgi?cmd

$=$ Retrieve $\& \mathrm{db}=$ PubMed\&list_uids $=14530753 \&$ dopt

$=$ Abstract

Block BM, Liu SS, Rowlingson AJ, et al. Efficacy of postoperative epidural analgesia: a meta-analysis. JAMA 2003;290:2455-63.

http://www.ncbi.nlm.nih.gov/entrez/query.fcgi?cmd

$=$ Retrieve $\& \mathrm{db}=$ PubMed\&list_uids $=$ 14612482\&dopt

$=$ Abstract
Boivin J. A review of psychosocial interventions in infertility. Soc Sci Med 2003;57:2325-41.

http://www.ncbi.nlm.nih.gov/entrez/query.fcgi?cmd

$=$ Retrieve $\& \mathrm{db}=$ PubMed\&list_uids = 14572840\&dopt

$=$ Abstract

Bollen CW, Uiterwaal CS, van Vught AJ. Cumulative metaanalysis of high-frequency versus conventional ventilation in premature neonates. Am J Respir Crit Care Med 2003; 168:1150-5.

http://www.ncbi.nlm.nih.gov/entrez/query.fcgi?cmd $=$ Retrieve \&db $=$ PubMed\&list_uids $=14607823 \&$ dopt

$=$ Abstract

Brown A, Taylor R, Noorani H, et al. A clinical and economic review of exercise-based cardiac rehabilitation programs for coronary artery disease. Ottawa: Canadian Coordinating Office for Health Technology Assessment (CCOHTA), August 2003.

www.ccohta.ca

Burke BL, Arkowitz H, Menchola M. The efficacy of motivational interviewing: a meta-analysis of controlled clinical trials. J Consult Clin Psychol 2003;71:843-61. http://www.ncbi.nlm.nih.gov/entrez/query.fcgi?cmd $=$ Retrieve $\& \mathrm{db}=$ PubMed\&list_uids $=14516234 \&$ dopt

$=$ Abstract

Burns KEA, Adhikari NKJ, Meade MO. Noninvasive positive pressure ventilation as a weaning strategy for intubated adults with respiratory failure. Cochrane Database Syst Rev 2003; (4):CD004127.

http://www.ncbi.nlm.nih.gov/entrez/query.fcgi?cmd

$=$ Retrieve $\& \mathrm{db}=$ PubMed\&list_uids $=14584005 \& \mathrm{dopt}$

$=$ Abstract

Carl JC, Myers TR, Kirchner HL, et al. Comparison of racemic albuterol and levalbuterol for treatment of acute asthma. J Pediatr 2003;143:731-6.

http://www.ncbi.nlm.nih.gov/entrez/query.fcgi?cmd

$=$ Retrieve \&db $=$ PubMed\&list_uids = 14657817\&dopt

$=$ Abstract

Cauley JA, Robbins J, Chen Z, et al. Effects of estrogen plus progestin on risk of fracture and bone mineral density: the Women's Health Initiative randomized trial. JAMA 2003;290: 1729-38.

http://www.ncbi.nlm.nih.gov/entrez/query.fcgi?cmd

$=$ Retrieve $\& \mathrm{db}=$ PubMed\&list_uids $=$ 14519707\&dopt

$=$ Abstract

Chastre J, Wolff M, Fagon JY, et al. Comparison of 8 vs 15 days of antibiotic therapy for ventilator-associated pneumonia in adults: a randomized trial. JAMA 2003;290:2588-98.

http://www.ncbi.nlm.nih.gov/entrez/query.fcgi?cmd

$=$ Retrieve $\& \mathrm{db}=$ PubMed\&list_uids $=$ 14625336\&dopt

$=$ Abstract 
2 of 14

Chaudhuri R, Livingston E, McMahon AD, et al. Cigarette smoking impairs the therapeutic response to oral corticosteroids in chronic asthma. Am J Respir Crit Care Med 2003; 168:1308-11.

http://www.ncbi.nlm.nih.gov/entrez/query.fcgi?cmd

$=$ Retrieve $\& \mathrm{db}=$ PubMed\&list uids $=12893649 \& \mathrm{dopt}$

$=$ Abstract

Chesney MA, Chambers DB, Taylor JM, et al. Coping effectiveness training for men living with HIV: results from a randomized clinical trial testing a group-based intervention. Psychosom Med 2003;65:1038-46.

http://www.ncbi.nlm.nih.gov/entrez/query.fcgi?cmd

$=$ Retrieve $\& \mathrm{db}=$ PubMed\&list_uids $=14645783 \&$ dopt

$=$ Abstract

Clarke MP, Wright CM, Hrisos S, et al. Randomised controlled trial of treatment of unilateral visual impairment detected at preschool vision screening. BMJ 2003;327:1251-5.

http://www.ncbi.nlm.nih.gov/entrez/query.fcgi?cmd

$=$ Retrieve $\& \mathrm{db}=$ PubMed\&list_uids $=$ 14644966\&dopt

$=$ Abstract

Cranney A, Waldegger L, Zytaruk N, et al. Risedronate for the prevention and treatment of postmenopausal osteoporosis. Cochrane Database Syst Rev 2003;(4):CD004523.

http://www.ncbi.nlm.nih.gov/entrez/query.fcgi?cmd

$=$ Retrieve $\& \mathrm{db}=$ PubMed\&list_uids $=14584020 \& \mathrm{dopt}$

$=$ Abstract

Crowther CA, Hiller JE, Doyle LW, et al. Effect of magnesium sulfate given for neuroprotection before preterm birth: a randomized controlled trial. JAMA 2003;290:2669-76. http://www.ncbi.nlm.nih.gov/entrez/query.fcgi?cmd

$=$ Retrieve $\& \mathrm{db}=$ PubMed\&list_uids = 14645308\&dopt

$=$ Abstract

Csonka P, Kaila M, Laippala P, et al. Oral prednisolone in the acute management of children age 6 to 35 months with viral respiratory infection-induced lower airway disease: a randomized, placebo-controlled trial. J Pediatr 2003;143:725-30.

http://www.ncbi.nlm.nih.gov/entrez/query.fcgi?cmd

$=$ Retrieve $\& \mathrm{db}=$ PubMed\&list_uids $=14657816 \& d o p t$

$=$ Abstract

Darlow BA, Austin NC. Selenium supplementation to prevent short-term morbidity in preterm neonates. Cochrane Database Syst Rev 2003;(4):CD003312.

http://www.ncbi.nlm.nih.gov/entrez/query.fcgi?cmd

$=$ Retrieve $\& \mathrm{db}=$ PubMed\&list_uids $=14583967 \&$ dopt

$=$ Abstract

Dear K, Holden J, Andrews R, et al. Vaccines for preventing pneumococcal infection in adults. Cochrane Database Syst Rev 2003; (4):CD000422.

http://www.ncbi.nlm.nih.gov/entrez/query.fcgi?cmd

$=$ Retrieve $\& \mathrm{db}=$ PubMed\&list_uids $=14583920 \& d o p t$

$=$ Abstract

de Jong Z, Munneke M, Zwinderman AH, et al. Is a long-term high-intensity exercise program effective and safe in patients with rheumatoid arthritis? Results of a randomized controlled trial. Arthritis Rheum 2003;48:2415-24.

http://www.ncbi.nlm.nih.gov/entrez/query.fcgi?cmd

$=$ Retrieve $\& \mathrm{db}=$ PubMed\&list_uids $=13130460 \& d o p t$

$=$ Abstract
Ehlers A, Clark DM, Hackmann A, et al. A randomized controlled trial of cognitive therapy, a self-help booklet, and repeated assessments as early interventions for posttraumatic stress disorder. Arch Gen Psychiatry 2003;60:1024-32.

http://www.ncbi.nlm.nih.gov/entrez/query.fcgi?cmd

$=$ Retrieve $\& \mathrm{db}=$ PubMed\&list uids $=14557148 \& \mathrm{dopt}$

$=$ Abstract

Ferrer M, Esquinas A, Leon $M$, et al. Noninvasive ventilation in severe hypoxemic respiratory failure: a randomized clinical trial. Am J Respir Crit Care Med 2003;168:1438-44.

http://www.ncbi.nlm.nih.gov/entrez/query.fcgi?cmd

$=$ Retrieve \&db $=$ PubMed\&list_uids $=14500259 \& d o p t$

$=$ Abstract

Fjaertoft H, Indredavik B, Lydersen S. Stroke unit care combined with early supported discharge: long-term followup of a randomized controlled trial. Stroke 2003;34:2687-91. http://www.ncbi.nlm.nih.gov/entrez/query.fcgi?cmd $=$ Retrieve \&db $=$ PubMed\&list_uids $=14576376 \& d o p t$

$=$ Abstract

Flenady VJ, Woodgate PG. Radiant warmers versus incubators for regulating body temperature in newborn infants. Cochrane Database Syst Rev 2003;(4):CD000435.

http://www.ncbi.nlm.nih.gov/entrez/query.fcgi?cmd

$=$ Retrieve $\& \mathrm{db}=$ PubMed\&list uids $=14583922 \& \mathrm{dopt}$

$=$ Abstract

Foley A, Halbert J, Hewitt T, et al. Does hydrotherapy improve strength and physical function in patients with osteoarthritis - a randomised controlled trial comparing a gym based and a hydrotherapy based strengthening programme. Ann Rheum Dis 2003;62:1162-7.

http://www.ncbi.nlm.nih.gov/entrez/query.fcgi?cmd

$=$ Retrieve $\& \mathrm{db}=$ PubMed\&list_uids $=14644853 \& \mathrm{dopt}$

$=$ Abstract

Francis CW, Berkowitz SD, Comp PC, et al. Comparison of ximelagatran with warfarin for the prevention of venous thromboembolism after total knee replacement. $N$ Engl J Med 2003;349:1703-12.

http://www.ncbi.nlm.nih.gov/entrez/query.fcgi?cmd $=$ Retrieve $\& \mathrm{db}=$ PubMed\&list_uids $=14585938 \& d o p t$

$=$ Abstract

Frerick H, Keitel W, Kuhn U, et al. Topical treatment of chronic low back pain with a capsicum plaster. Pain 2003; 106:59-64.

http://www.ncbi.nlm.nih.gov/entrez/query.fcgi?cmd $=$ Retrieve $\& \mathrm{db}=$ PubMed\&list uids $=14581111 \& \mathrm{dopt}$

$=$ Abstract

Frich LM. Nursing interventions for patients with chronic conditions. J Adv Nurs 2003;44:137-53.

http://www.ncbi.nlm.nih.gov/entrez/query.fcgi?cmd

$=$ Retrieve $\& \mathrm{db}=$ PubMed\&list_uids $=14521681 \& d o p t$

$=$ Abstract

Frosch DL, Kaplan RM, Felitti VJ. A randomized controlled trial comparing internet and video to facilitate patient education for men considering the prostate specific antigen test. J Gen Intern Med 2003; 18:781-7.

http://www.ncbi.nlm.nih.gov/entrez/query.fcgi?cmd $=$ Retrieve $\& \mathrm{db}=$ PubMed\&list_uids $=14521639 \& d o p t$

$=$ Abstract 
Gardner JM, Walker SP, Powell CA, et al. A randomized controlled trial of a home-visiting intervention on cognition and behavior in term low birth weight infants. $J$ Pediatr 2003; 143:634-9.

http://www.ncbi.nlm.nih.gov/entrez/query.fcgi?cmd

$=$ Retrieve $\& \mathrm{db}=$ PubMed\&list_uids = 14615736\&dopt

$=$ Abstract

Garrel D, Patenaude J, Nedelec B, et al. Decreased mortality and infectious morbidity in adult burn patients given enteral glutamine supplements: a prospective, controlled, randomized clinical trial. Crit Care Med 2003;31:2444-9.

http://www.ncbi.nlm.nih.gov/entrez/query.fcgi?cmd

$=$ Retrieve $\& \mathrm{db}=$ PubMed\&list_uids $=14530749 \& \mathrm{dopt}$

$=$ Abstract

Gillespie L, Gillespie W, Robertson M, et al. Interventions for preventing falls in elderly people. Cochrane Database Syst Rev 2003; (4):CD000340.

http://www.ncbi.nlm.nih.gov/entrez/query.fcgi?cmd

$=$ Retrieve $\& \mathrm{db}=$ PubMed\&list_uids $=14583918 \& \mathrm{dopt}$

$=$ Abstract

Gillies D, O'Riordan L, Carr D, et al. Gauze and tape and transparent polyurethane dressings for central venous catheters. Cochrane Database Syst Rev 2003;(4):CD003827.

http://www.ncbi.nlm.nih.gov/entrez/query.fcgi?cmd

$=$ Retrieve $\& \mathrm{db}=$ PubMed\&list_uids $=14583995 \&$ dopt

$=$ Abstract

Ginzburg E, Cohn SM, Lopez J, et al. Randomized clinical trial of intermittent pneumatic compression and low molecular weight heparin in trauma. Br J Surg 2003;90:1338-44.

http://www.ncbi.nlm.nih.gov/entrez/query.fcgi?cmd

$=$ Retrieve $\& \mathrm{db}=$ PubMed\&list_uids = 14598411\&dopt

$=$ Abstract

Giraudet-Le Quintrec JS, Coste J, Vastel L, et al. Positive effect of patient education for hip surgery: a randomized trial. Clin Orthop 2003;414:112-20.

http://www.ncbi.nlm.nih.gov/entrez/query.fcgi?cmd

$=$ Retrieve $\& \mathrm{db}=$ PubMed\&list_uids = 12966284\&dopt

$=$ Abstract

Gisbert JP, Khorrami S, Calvet X, et al. Meta-analysis: proton pump inhibitors vs. $\mathrm{H}_{2}$-receptor antagonists-their efficacy with antibiotics in Helicobacter pylori eradication. Aliment Pharmacol Ther 2003;18:757-66.

http://www.ncbi.nlm.nih.gov/entrez/query.fcgi?cmd

$=$ Retrieve \&db $=$ PubMed\&list_uids = 14535868\&dopt

$=$ Abstract

Glazener CMA, Evans JHC, Peto RE. Drugs for nocturnal enuresis in children (other than desmopressin and tricyclics). Cochrane Database Syst Rev 2003;(4):CD002238.

http://www.ncbi.nlm.nih.gov/entrez/query.fcgi?cmd

$=$ Retrieve \&db $=$ PubMed\&list_uids $=$ 14583948\&dopt

$=$ Abstract

Graffy J, Taylor J, Williams A, et al. Randomised controlled trial of support from volunteer counsellors for mothers considering breast feeding. BMJ 2004;328:26-31.

http://www.ncbi.nlm.nih.gov/entrez/query.fcgi?cmd

$=$ Retrieve $\& \mathrm{db}=$ PubMed\&list_uids $=14703543 \&$ dopt

$=$ Abstract

Haddock G, Barrowclough C, Tarrier N, et al. Cognitivebehavioural therapy and motivational intervention for schizophrenia and substance misuse. 18-month outcomes of a randomised controlled trial. Br J Psychiatry 2003;183:41826.

http://www.ncbi.nlm.nih.gov/entrez/query.fcgi?cmd

$=$ Retrieve $\& \mathrm{db}=$ PubMed\&list_uids $=14594917 \& \mathrm{dopt}$

$=$ Abstract

Hailey D, Jacobs P, Mayers I, et al. Auto-titrating nasal continuous positive airway pressure systems in the management of obstructive sleep apnea. Ottawa: Canadian Coordinating Office for Health Technology Assessment (CCOHTA), September 2003.

www.ccohta.ca

Higgins ST, Sigmon SC, Wong CJ, et al. Community reinforcement therapy for cocaine-dependent outpatients. Arch Gen Psychiatry 2003;60:1043-52.

http://www.ncbi.nlm.nih.gov/entrez/query.fcgi?cmd

$=$ Retrieve $\& \mathrm{db}=$ PubMed\&list_uids $=$ 14557150\&dopt

$=$ Abstract

Hovell MF, Sipan CL, Blumberg EJ, et al. Increasing Latino adolescents' adherence to treatment for latent tuberculosis infection: a controlled trial. Am J Public Health 2003;93:1871-7. http://www.ncbi.nlm.nih.gov/entrez/query.fcgi?cmd

$=$ Retrieve $\& \mathrm{db}=$ PubMed\&list_uids $=14600055 \& \mathrm{dopt}$

$=$ Abstract

Husereau D, Clifford T, Aker P, et al. Spinal manipulation for infantile colic. Ottawa: Canadian Coordinating Office for Health Technology Assessment (CCOHTA), November 2003. www.ccohta.ca

Janson SL, Fahy JV, Covington JK, et al. Effects of individual self-management education on clinical, biological, and adherence outcomes in asthma. Am J Med 2003;115:620-6. http://www.ncbi.nlm.nih.gov/entrez/query.fcgi?cmd $=$ Retrieve $\& \mathrm{db}=$ PubMed\&list_uids = 14656614\&dopt

$=$ Abstract

Jewell D, Young G. Interventions for nausea and vomiting in early pregnancy. Cochrane Database Syst Rev 2003; (4):CD000145.

http://www.ncbi.nlm.nih.gov/entrez/query.fcgi?cmd

$=$ Retrieve\&db = PubMed\&list uids = 14583914\&dopt

$=$ Abstract

Johnson A, Sandford J, Tyndall J. Written and verbal information versus verbal informal information only for patients being discharged from acute hospital setting to home. Cochrane Database Syst Rev 2003;(4):CD003716. http://www.ncbi.nlm.nih.gov/entrez/query.fcgi?cmd $=$ Retrieve $\& \mathrm{db}=$ PubMed\&list_uids $=14583990 \& \mathrm{dopt}$ $=$ Abstract

Jordan KM, Arden NK, Doherty M, et al. EULAR recommendations 2003: an evidence based approach to the management of knee osteoarthritis: report of a Task Force of the Standing Committee for International Clinical Studies Including Therapeutic Trials (ESCISIT). Ann Rheum Dis 2003;62:1145-55.

http://www.ncbi.nlm.nih.gov/entrez/query.fcgi?cmd $=$ Retrieve $\& \mathrm{db}=$ PubMed\&list_uids $=14644851 \&$ dopt

$=$ Abstract

Jorge RE, Robinson RG, Arndt S, et al. Mortality and poststroke depression: a placebo-controlled trial of antidepressants. Am J Psychiatry 2003;160:1823-9. 
4 of 14

http://www.ncbi.nlm.nih.gov/entrez/query.fcgi?cmd

$=$ Retrieve \&db $=$ PubMed\&list_uids $=14514497 \& d o p t$

$=$ Abstract

Kalani M, Apelqvist J, Blombäck M, et al. Effect of dalteparin on healing of chronic foot ulcers in diabetic patients with peripheral arterial occlusive disease: a prospective, randomized, double-blind, placebo-controlled study. Diabetes Care 2003;26:2575-80.

http://www.ncbi.nlm.nih.gov/entrez/query.fcgi?cmd

$=$ Retrieve $\& \mathrm{db}=$ PubMed\&list_uids = 12941721\&dopt

$=$ Abstract

Kamps AW, Brand PL, Kimpen JL, et al. Outpatient management of childhood asthma by paediatrician or asthma nurse: randomised controlled study with one year follow up. Thorax 2003;58:968-73.

http://www.ncbi.nlm.nih.gov/entrez/query.fcgi?cmd

$=$ Retrieve $\& \mathrm{db}=$ PubMed\&list_uids $=14586050 \& \mathrm{dopt}$

$=$ Abstract

Kaur G, Gupta P, Kumar A. A randomized trial of eutectic mixture of local anesthetics during lumbar puncture in newborns. Arch Pediatr Adolesc Med 2003;157:1065-70.

http://www.ncbi.nlm.nih.gov/entrez/query.fcgi?cmd

$=$ Retrieve \&db $=$ PubMed\&list_uids $=14609894 \& d o p t$

$=$ Abstract

Koebnick C, Wagner I, Leitzmann P, et al. Probiotic beverage containing Lactobacillus casei Shirota improves gastrointestinal symptoms in patients with chronic constipation. Can J Gastroenterol 2003;17:655-9.

http://www.ncbi.nlm.nih.gov/entrez/query.fcgi?cmd

$=$ Retrieve $\& \mathrm{db}=$ PubMed\&list_uids $=14631461 \&$ dopt

$=$ Abstract

Kolbach D, Sandbrink M, Neumann H, et al. Compression therapy for treating stage I and II (Widmer) post-thrombotic syndrome. Cochrane Database Syst Rev 2003;(4):CD004177.

http://www.ncbi.nlm.nih.gov/entrez/query.fcgi?cmd

$=$ Retrieve $\& \mathrm{db}=$ PubMed\&list_uids $=14584008 \& \mathrm{dopt}$

$=$ Abstract

Kovacs FM, Abraira V, Peña A, et al. Effect of firmness of mattress on chronic non-specific low-back pain: randomised, double-blind, controlled, multicentre trial. Lancet 2003;362: 1599-604.

http://www.ncbi.nlm.nih.gov/entrez/query.fcgi?cmd

$=$ Retrieve $\& \mathrm{db}=$ PubMed\&list_uids $=14630439 \& \mathrm{dopt}$

$=$ Abstract

Lepore SJ, Helgeson VS, Eton DT, et al. Improving quality of life in men with prostate cancer: a randomized controlled trial of group education interventions. Health Psychol 2003;22:443-52.

http://www.ncbi.nlm.nih.gov/entrez/query.fcgi?cmd

$=$ Retrieve $\& \mathrm{db}=$ PubMed\&list_uids $=14570527 \& \mathrm{dopt}$

$=$ Abstract

Lim E, Ali Z, Ali A, et al. Indirect comparison meta-analysis of aspirin therapy after coronary surgery. BMJ 2003;327:130913.

http://www.ncbi.nlm.nih.gov/entrez/query.fcgi?cmd

$=$ Retrieve $\& \mathrm{db}=$ PubMed\&list_uids $=$ 14656836\&dopt

$=$ Abstract

Lin EH, Katon W, Von Korff M, et al. Effect of improving depression care on pain and functional outcomes among older adults with arthritis: a randomized controlled trial. JAMA 2003;290:2428-9.

http:/www.ncbi.nlm.nih.gov/entrez/query.fcgi?cmd

$=$ Retrieve $\& \mathrm{db}=$ PubMed\&list_uids $=14612479 \& \mathrm{dopt}$

$=$ Abstract

Loeb M, Main C, Walker-Dilks C, et al. Antimicrobial drugs for treating methicillin-resistant Staphylococcus aureus colonization. Cochrane Database Syst Rev 2003;(4): CD003340.

http://www.ncbi.nlm.nih.gov/entrez/query.fcgi?cmd $=$ Retrieve $\& \mathrm{db}=$ PubMed\&list_uids $=14583969 \& \mathrm{dopt}$

$=$ Abstract

Lord SR, Castell S, Corcoran J, et al. The effect of group exercise on physical functioning and falls in frail older people living in retirement villages: a randomized, controlled trial. J Am Geriatr Soc 2003;51:1685-92.

http://www.ncbi.nlm.nih.gov/entrez/query.fcgi?cmd

$=$ Retrieve $\& \mathrm{db}=$ PubMed\&list_uids $=14687345 \& \mathrm{dopt}$

$=$ Abstract

Lubetsky A, Yonath H, Olchovsky D, et al. Comparison of oral vs intravenous phytonadione (vitamin Kl) in patients with excessive anticoagulation: a prospective randomized controlled study. Arch Intern Med 2003;163: 2469-73.

http://www.ncbi.nlm.nih.gov/entrez/query.fcgi?cmd

$=$ Retrieve $\& \mathrm{db}=$ PubMed\&list_uids $=14609783 \& \mathrm{dopt}$

$=$ Abstract

MacKelvie KJ, Khan KM, Petit MA, et al. A school-based exercise intervention elicits substantial bone health benefits: a 2-year randomized controlled trial in girls. Pediatrics 2003;112:e447-52.

http://www.ncbi.nlm.nih.gov/entrez/query.fcgi?cmd

$=$ Retrieve $\& \mathrm{db}=$ PubMed\&list_uids $=14654643 \& \mathrm{dopt}$

$=$ Abstract

Macleod ZR, Charles MA, Arnaldi VC, et al. Telephone counselling as an adjunct to nicotine patches in smoking cessation: a randomised controlled trial. Med $J$ Aust 2003; 179:349-52.

http:/www.ncbi.nlm.nih.gov/entrez/query.fcgi?cmd

$=$ Retrieve $\& \mathrm{db}=$ PubMed\&list_uids $=14503897 \&$ dopt

$=$ Abstract

Marjoribanks J, Proctor ML, Farquhar C. Nonsteroidal antiinflammatory drugs for primary dysmenorrhoea. Cochrane Database Syst Rev 2003;(4):CD001751.

http://www.ncbi.nlm.nih.gov/entrez/query.fcgi?cmd

$=$ Retrieve\&db $=$ PubMed\&list_uids = 14583938\&dopt

$=$ Abstract

McElhaney JE, Gravenstein S, Cole SK, et al. A placebocontrolled trial of a proprietary extract of North American ginseng (CVT-E002) to prevent acute respiratory illness in institutionalized older adults. $J$ Am Geriatr Soc 2004;52:13-9.

http://www.ncbi.nlm.nih.gov/entrez/query.fcgi?cmd $=$ Retrieve \&db $=$ PubMed\&list_uids $=14687309 \& d o p t$

$=$ Abstract

Mitchell M. Patient anxiety and modern elective surgery: a literature review. J Clin Nurs 2003;12:806-15.

http://www.ncbi.nlm.nih.gov/entrez/query.fcgi?cmd

$=$ Retrieve $\& \mathrm{db}=$ PubMed\&list_uids $=14632973 \& \mathrm{dopt}$

$=$ Abstract 
5 of 14

Moayyedi P, Deeks J, Talley NJ, et al. An update of the Cochrane systematic review of Helicobacter pylori eradication therapy in nonulcer dyspepsia: resolving the discrepancy between systematic reviews. Am $J$ Gastroenterol 2003;98:2621-6.

http://www.ncbi.nlm.nih.gov/entrez/query.fcgi?cmd $=$ Retrieve $\& \mathrm{db}=$ PubMed\&list_uids $=14687807 \& d o p t$ $=$ Abstract

Nava S, Carbone G, DiBattista $\mathrm{N}$, et al. Noninvasive ventilation in cardiogenic pulmonary edema: a multicenter randomized trial. Am J Respir Crit Care Med 2003; 168:1432-7.

http://www.ncbi.nlm.nih.gov/entrez/query.fcgi?cmd $=$ Retrieve $\& \mathrm{db}=$ PubMed\&list_uids $=12958051 \& \mathrm{dopt}$

$=$ Abstract

Nett R, Landy S, Shackelford S, et al. Pain-free efficacy after treatment with sumatriptan in the mild pain phase of menstrually associated migraine. Obstet Gynecol 2003; 102:835-42.

http://www.ncbi.nlm.nih.gov/entrez/query.fcgi?cmd

$=$ Retrieve $\& \mathrm{db}=$ PubMed\&list_uids $=14551015 \& \mathrm{dopt}$

$=$ Abstract

Nezu AM, Nezu CM, Felgoise SH, et al. Project Genesis: assessing the efficacy of problem-solving therapy for distressed adult cancer patients. J Consult Clin Psychol 2003. 71:1036-48

http://www.ncbi.nlm.nih.gov/entrez/query.fcgi?cmd

$=$ Retrieve $\& \mathrm{db}=$ PubMed\&list_uids $=14622079 \& \mathrm{dopt}$

$=$ Abstract

Nilsson L, Kjellman NI, Bjorksten B. Allergic disease at the age of 7 years after pertussis vaccination in infancy: results from the follow-up of a randomized controlled trial of 3 vaccines. Arch Pediatr Adolesc Med 2003;157:1184-9.

http://www.ncbi.nlm.nih.gov/entrez/query.fcgi?cmd

$=$ Retrieve $\& \mathrm{db}=$ PubMed\&list_uids $=14662571 \& \mathrm{dopt}$

$=$ Abstract

Noorani HZ, Brady B, McGahan L, et al. A clinical and economic review of stroke rehabilitation services. Ottawa: Canadian Coordinating Office for Health and Technology Assessment (CCOHTA), August 2003.

www.ccohta.ca

Norton C, Chelvanayagam S, Wilson-Barnett J, et al. Randomized controlled trial of biofeedback for fecal incontinence. Gastroenterology 2003;125:1320-9.

http://www.ncbi.nlm.nih.gov/entrez/query.fcgi?cmd

$=$ Retrieve \&db $=$ PubMed\&list_uids = 14598248\&dopt

$=$ Abstract

Oates-Whitehead RM, D'Angelo A, Mol B. Anticoagulant and aspirin prophylaxis for preventing thromboembolism after major gynaecological surgery. Cochrane Database Syst Rev 2003; (4):CD003679.

http://www.ncbi.nlm.nih.gov/entrez/query.fcgi?cmd

$=$ Retrieve $\& \mathrm{db}=$ PubMed\&list_uids $=14583989 \& d o p t$

$=$ Abstract

Oh H, Seo W. A meta-analysis of the effects of various interventions in preventing endotracheal suction-induced hypoxemia. J Clin Nurs 2003;12:912-24.

http://www.ncbi.nlm.nih.gov/entrez/query.fcgi?cmd

$=$ Retrieve $\& \mathrm{db}=$ PubMed\&list_uids $=14632984 \& \mathrm{dopt}$

$=$ Abstract
O'Mathuna DP, Ashford RL. Therapeutic touch for healing acute wounds. Cochrane Database Syst Rev 2003;(4):CD002766. http://www.ncbi.nlm.nih.gov/entrez/query.fcgi?cmd

$=$ Retrieve $\& \mathrm{db}=$ PubMed\&list_uids $=14583953 \&$ dopt

$=$ Abstract

Owen CG, Whincup PH, Gilg JA, et al. Effect of breast feeding in infancy on blood pressure in later life: systematic review and meta-analysis. BMJ 2003;327:1189-95.

http://www.ncbi.nlm.nih.gov/entrez/query.fcgi?cmd

$=$ Retrieve $\& \mathrm{db}=$ PubMed\&list_uids $=14630752 \& \mathrm{dopt}$

$=$ Abstract

Pharoah FM, Rathbone J, Mari JJ, et al. Family intervention for schizophrenia. Cochrane Database Syst Rev 2003;(4):CD000088.

http://www.ncbi.nlm.nih.gov/entrez/query.fcgi?cmd

$=$ Retrieve $\& \mathrm{db}=$ PubMed\&list uids $=14583908 \& \mathrm{dopt}$

$=$ Abstract

Pike KM, Walsh BT, Vitousek K, et al. Cognitive behavior therapy in the posthospitalization treatment of anorexia nervosa. Am J Psychiatry 2003;160:2046-9.

http://www.ncbi.nlm.nih.gov/entrez/query.fcgi?cmd

$=$ Retrieve $\& \mathrm{db}=$ PubMed\&list_uids $=14594754 \&$ dopt

$=$ Abstract

Ram FSF, Wedzicha JA, Wright J, et al. Hospital at home for acute exacerbations of chronic obstructive pulmonary disease. Cochrane Database Syst Rev 2003;(4):CD003573.

http://www.ncbi.nlm.nih.gov/entrez/query.fcgi?cmd

$=$ Retrieve \&db = PubMed\&list_uids = 14583984\&dopt

$=$ Abstract

Rashid P, Leonardi-Bee J, Bath P. Blood pressure reduction and secondary prevention of stroke and other vascular events: a systematic review. Stroke 2003;34:2741-8. http://www.ncbi.nlm.nih.gov/entrez/query.fcgi?cmd $=$ Retrieve\&db = PubMed\&list uids = 14576382\&dopt

$=$ Abstract

Redmon JB, Raatz SK, Reck KP, et al. One-year outcome of a combination of weight loss therapies for subjects with type 2 diabetes: a randomized trial. Diabetes Care 2003;26:2505-11. http://www.ncbi.nlm.nih.gov/entrez/query.fcgi?cmd $=$ Retrieve \&db = PubMed\&list_uids = 12941710\&dopt $=$ Abstract

Reis EC, Roth EK, Syphan JL, et al. Effective pain reduction for multiple immunization injections in young infants. Arch Pediatr Adolesc Med 2003;157:1115-20.

http://www.ncbi.nlm.nih.gov/entrez/query.fcgi?cmd

$=$ Retrieve\&db = PubMed\&list_uids = 14609903\&dopt

$=$ Abstract

Salpeter SR, Greyber E, Pasternak GA, et al. Risk of fatal and nonfatal lactic acidosis with metformin use in type 2 diabetes mellitus: systematic review and meta-analysis. Arch Intern Med 2003;163:2594-602.

http://www.ncbi.nlm.nih.gov/entrez/query.fcgi?cmd

$=$ Retrieve $\& \mathrm{db}=$ PubMed\&list_uids $=14638559 \& \mathrm{dopt}$

$=$ Abstract

Satterfield DW, Volansky M, Caspersen CJ, et al. Communitybased lifestyle interventions to prevent type 2 diabetes. Diabetes Care 2003;26:2643-52.

http:/www.ncbi.nlm.nih.gov/entrez/query.fcgi?cmd

$=$ Retrieve $\& \mathrm{db}=$ PubMed\&list_uids $=12941733 \& \mathrm{dopt}$

$=$ Abstract 
6 of 14

Schlagenhauf P, Tschopp A, Johnson R, et al. Tolerability of malaria chemoprophylaxis in non-immune travellers to subSaharan Africa: multicentre, randomised, double blind, four arm study. BMJ 2003;327:1078-83.

http://www.ncbi.nlm.nih.gov/entrez/query.fcgi?cmd

$=$ Retrieve $\& \mathrm{db}=$ PubMed\&list_uids $=14604928 \& \mathrm{dopt}$

$=$ Abstract

Schreiber MD, Gin-Mestan K, Marks JD, et al. Inhaled nitric oxide in premature infants with the respiratory distress syndrome. N Engl J Med 2003;349:2099-107.

http://www.ncbi.nlm.nih.gov/entrez/query.fcgi?cmd

$=$ Retrieve \&db $=$ PubMed\&list_uids $=14645637 \& d o p t$

$=$ Abstract

Shah V, Taddio A, Kulasekaran K, et al. Evaluation of a new lancet device (BD QuikHeel) on pain response and success of procedure in term neonates. Arch Pediatr Adolesc Med 2003; 157:1075-8.

http://www.ncbi.nlm.nih.gov/entrez/query.fcgi?cmd

$=$ Retrieve \&db $=$ PubMed\&list_uids $=14609896 \& d o p t$

$=$ Abstract

Shekelle P, Morton SC, Hardy M, et al. Effect of supplemental antioxidants vitamin $C$, vitamin $E$, and coenzyme $Q 10$ for the prevention and treatment of cardiovascular disease. Evidence Report/Technology Assessment Number 83. AHRQ Publication No. 03-0043. Rockville, MD: Agency for Healthcare Research and Quality, July 2003.

www.ahrq.gov

Simons SH, van Dijk M, van Lingen RA, et al. Routine morphine infusion in preterm newborns who received ventilatory support: a randomized controlled trial. JAMA 2003;290:2419-27.

http://www.ncbi.nlm.nih.gov/entrez/query.fcgi?cmd

$=$ Retrieve $\& \mathrm{db}=$ PubMed\&list_uids $=14612478 \& \mathrm{dopt}$

$=$ Abstract

Sok SR, Erlen JA, Kim KB. Effects of acupuncture therapy on insomnia. $J$ Adv Nurs 2003;44:375-84.

http://www.ncbi.nlm.nih.gov/entrez/query.fcgi?cmd

$=$ Retrieve $\& \mathrm{db}=$ PubMed\&list_uids $=14651709 \& \mathrm{dopt}$

$=$ Abstract

Spooner $\mathrm{CH}$, Spooner GR, Rowe RH. Mast-cell stabilizing agents to prevent exercise-induced bronchoconstriction. Cochrane Database Syst Rev 2003;(4):CD002307.

http://www.ncbi.nlm.nih.gov/entrez/query.fcgi?cmd

$=$ Retrieve $\& \mathrm{db}=$ PubMed\&list_uids $=14583951 \& \mathrm{dopt}$

$=$ Abstract

Stacey D, Noorani HZ, Fisher A, et al. Telephone triage services: systematic review and a survey of Canadian call centre programs. Ottawa: Canadian Coordinating Office for Health Technology Assessment, November 2003.

www.ccohta.ca

Staiger TO, Gaster B, Sullivan MD, et al. Systematic review of antidepressants in the treatment of chronic low back pain. Spine 2003;28:2540-5.

http://www.ncbi.nlm.nih.gov/entrez/query.fcgi?cmd

$=$ Retrieve $\& \mathrm{db}=$ PubMed\&list_uids $=14624092 \& \mathrm{dopt}$

$=$ Abstract

Stefanescu BM, Murphy WP, Hansell BJ, et al. A randomized, controlled trial comparing two different continuous positive airway pressure systems for the successful extubation of extremely low birth weight infants. Pediatrics 2003;112: 1031-8.

http://www.ncbi.nlm.nih.gov/entrez/query.fcgi?cmd

$=$ Retrieve $\& \mathrm{db}=$ PubMed\&list_uids $=14595042 \& \mathrm{dopt}$

$=$ Abstract

Stephenson N, Dalton JA, Carlson J. The effect of foot reflexology on pain in patients with metastatic cancer. Appl Nurs Res 2003;16:284-6.

http://www.ncbi.nlm.nih.gov/entrez/query.fcgi?cmd

$=$ Retrieve $\& \mathrm{db}=$ PubMed\&list_uids $=14608562 \&$ dopt

$=$ Abstract

Sustained effect of intensive treatment of type 1 diabetes mellitus on development and progression of diabetic nephropathy: the Epidemiology of Diabetes Interventions and Complications (EDIC) study. JAMA 2003;290:2159-67.

http://www.ncbi.nlm.nih.gov/entrez/query.fcgi?cmd

$=$ Retrieve $\& \mathrm{db}=$ PubMed\&list_uids $=14570951 \& \mathrm{dopt}$

$=$ Abstract

Sutherland ER, Allmers H, Ayas NT, et al. Inhaled corticosteroids reduce the progression of airflow limitation in chronic obstructive pulmonary disease: a meta-analysis. Thorax 2003;58:937-41.

http://www.ncbi.nlm.nih.gov/entrez/query.fcgi?cmd

$=$ Retrieve\&db = PubMed\&list_uids = 14586043\&dopt

$=$ Abstract

Swan GE, McAfee T, Curry SJ, et al. Effectiveness of bupropion sustained release for smoking cessation in a health care setting: a randomized trial. Arch Intern Med 2003;163:2337-44. http://www.ncbi.nlm.nih.gov/entrez/query.fcgi?cmd

$=$ Retrieve $\& \mathrm{db}=$ PubMed\&list_uids = 14581254\&dopt

$=$ Abstract

Swenson JR, O'Connor CM, Barton D, et al. Influence of depression and effect of treatment with sertraline on quality of life after hospitalization for acute coronary syndrome. Am J Cardiol 2003;92:1271-6.

http://www.ncbi.nlm.nih.gov/entrez/query.fcgi?cmd

$=$ Retrieve $\& \mathrm{db}=$ PubMed\&list_uids $=14636902 \& \mathrm{dopt}$

$=$ Abstract

Swingler G, Fransman D, Hussey G. Conjugate vaccines for preventing Haemophilus influenzae type b infections. Cochrane Database Syst Rev 2003;(4):CD001729.

http://www.ncbi.nlm.nih.gov/entrez/query.fcgi?cmd

$=$ Retrieve $\& \mathrm{db}=$ PubMed\&list_uids $=14583937 \& \mathrm{dopt}$

$=$ Abstract

Symington A, Pinelli J. Developmental care for promoting development and preventing morbidity in preterm infants. Cochrane Database Syst Rev 2003;(4):CD001814.

http://www.ncbi.nlm.nih.gov/entrez/query.fcgi?cmd

$=$ Retrieve $\& \mathrm{db}=$ PubMed\&list_uids $=14583939 \& d o p t$

$=$ Abstract

Taramarcaz P, Gibson PG. Intranasal corticosteroids for asthma control in people with coexisting asthma and rhinitis. Cochrane Database Syst Rev 2003;(4):CD003570.

http://www.ncbi.nlm.nih.gov/entrez/query.fcgi?cmd

$=$ Retrieve $\& \mathrm{db}=$ PubMed\&list_uids $=14583983 \& \mathrm{dopt}$

$=$ Abstract

Taubman B, Blum NJ, Nemeth N. Stool toileting refusal: a prospective intervention targeting parental behavior. Arch Pediatr Adolesc Med 2003;157:1193-6. 
http://www.ncbi.nlm.nih.gov/entrez/query.fcgi?cmd $=$ Retrieve $\& \mathrm{db}=$ PubMed\&list_uids $=14662573 \& \mathrm{dopt}$ $=$ Abstract

Teri L, Gibbons LE, McCurry SM, et al. Exercise plus behavioral management in patients with Alzheimer disease: a randomized controlled trial. JAMA 2003;290:2015-22. http://www.ncbi.nlm.nih.gov/entrez/query.fcgi?cmd $=$ Retrieve $\& \mathrm{db}=$ PubMed\&list_uids $=14559955 \& \mathrm{dopt}$ $=$ Abstract

Toroyan T, Roberts I, Oakley A, et al. Effectiveness of out-ofhome day care for disadvantaged families: randomised controlled trial. BMJ 2003;327:906-10.

http://www.ncbi.nlm.nih.gov/entrez/query.fcgi?cmd

$=$ Retrieve $\& \mathrm{db}=$ PubMed\&list_uids $=14563749 \& d o p t$

$=$ Abstract

Turnbull F. Effects of different blood-pressure-lowering regimens on major cardiovascular events: results of prospectively-designed overviews of randomised trials. Lancet 2003;362:1527-35.

http://www.ncbi.nlm.nih.gov/entrez/query.fcgi?cmd

$=$ Retrieve $\& \mathrm{db}=$ PubMed\&list_uids $=14615107 \& \mathrm{dopt}$

$=$ Abstract

Vakil N, Fennerty MB. Direct comparative trials of the efficacy of proton pump inhibitors in the management of gastro-oesophageal reflux disease and peptic ulcer disease. Aliment Pharmacol Ther 2003;18:559-68.

http://www.ncbi.nlm.nih.gov/entrez/query.fcgi?cmd

$=$ Retrieve $\& \mathrm{db}=$ PubMed\&list_uids $=12969082 \& \mathrm{dopt}$

$=$ Abstract

van Alem AP, Vrenken RH, de Vos R, et al. Use of automated external defibrillator by first responders in out of hospital cardiac arrest: prospective controlled trial. BMJ 2003;327:1312-16.

http://www.ncbi.nlm.nih.gov/entrez/query.fcgi?cmd

$=$ Retrieve $\& \mathrm{db}=$ PubMed\&list_uids $=14656837 \& d o p t$

$=$ Abstract

van Empelen P, Kok G, van Kesteren NM, et al. Effective methods to change sex-risk among drug users: a review of psychosocial interventions. Soc Sci Med 2003;57:1593-608.

http://www.ncbi.nlm.nih.gov/entrez/query.fcgi?cmd

$=$ Retrieve \&db $=$ PubMed\&list_uids $=12948569 \&$ dopt

$=$ Abstract

van Pinxteren B, Numans ME, Lau J, et al. Short-term treatment of gastroesophageal reflux disease. J Gen Intern Med 2003; 18:755-63.

http://www.ncbi.nlm.nih.gov/entrez/query.fcgi?cmd

$=$ Retrieve $\& \mathrm{db}=$ PubMed\&list uids $=12950485 \&$ dopt

$=$ Abstract

Warren JM, Henry CJ, Simonite V. Low glycemic index breakfasts and reduced food intake in preadolescent children. Pediatrics 2003;112:e414-9.

http://www.ncbi.nlm.nih.gov/entrez/query.fcgi?cmd

$=$ Retrieve \&db $=$ PubMed\&list_uids $=14595085 \&$ dopt

$=$ Abstract

Watson CP, Moulin D, Watt-Watson J, et al. Controlledrelease oxycodone relieves neuropathic pain: a randomized controlled trial in painful diabetic neuropathy. Pain 2003;105:71-8 http://www.ncbi.nlm.nih.gov/entrez/query.fcgi?cmd

$=$ Retrieve $\& \mathrm{db}=$ PubMed\&list_uids $=14499422 \&$ dopt

$=$ Abstract

Weintrob N, Benzaquen H, Galatzer A, et al. Comparison of continuous subcutaneous insulin infusion and multiple daily injection regimens in children with type l diabetes: a randomized open crossover trial. Pediatrics 2003;112:559-64. http://www.ncbi.nlm.nih.gov/entrez/query.fcgi?cmd $=$ Retrieve $\& \mathrm{db}=$ PubMed\&list_uids $=12949284 \& \mathrm{dopt}$

$=$ Abstract

Weiss SR, Cheng SL, Kourides IA, et al. Inhaled insulin provides improved glycemic control in patients with type 2 diabetes mellitus inadequately controlled with oral agents: a randomized controlled trial. Arch Intern Med 2003; 163:2277-82.

http://www.ncbi.nlm.nih.gov/entrez/query.fcgi?cmd

$=$ Retrieve $\& \mathrm{db}=$ PubMed\&list_uids $=14581245 \& \mathrm{dopt}$

$=$ Abstract

Whatley VN, Dodds CL, Paul RI. Randomized clinical trial of docusate, triethanolamine polypeptide, and irrigation in cerumen removal in children. Arch Pediatr Adolesc Med 2003; 157:1177-80.

http://www.ncbi.nlm.nih.gov/entrez/query.fcgi?cmd

$=$ Retrieve $\& \mathrm{db}=$ PubMed\&list_uids $=14662569 \& \mathrm{dopt}$

$=$ Abstract

Wiffen PJ, Edwards JE, Barden J, et al. Oral morphine for cancer pain. Cochrane Database Syst Rev 2003;(4):CD003868.

http://www.ncbi.nlm.nih.gov/entrez/query.fcgi?cmd

$=$ Retrieve $\& \mathrm{db}=$ PubMed\&list_uids $=14583997 \&$ dopt

$=$ Abstract

Wilken JA, Kane RL, Ellis AK, et al. A comparison of the effect of diphenhydramine and desloratadine on vigilance and cognitive function during treatment of ragweedinduced allergic rhinitis. Ann Allergy Asthma Immunol 2003;91:375-85.

http://www.ncbi.nlm.nih.gov/entrez/query.fcgi?cmd

$=$ Retrieve $\& \mathrm{db}=$ PubMed\&list_uids $=14582817 \& \mathrm{dopt}$

$=$ Abstract

Wille-Jorgensen $\mathrm{P}$, Rasmussen MS, Andersen BR, et al. Heparins and mechanical methods for thromboprophylaxis in colorectal surgery. Cochrane Database Syst Rev 2004; (4):CD001217.

http://www.ncbi.nlm.nih.gov/entrez/query.fcgi?cmd

$=$ Retrieve $\& \mathrm{db}=$ PubMed\&list_uids $=14583929 \& \mathrm{dopt}$

$=$ Abstract

Williams ME, Pulliam CC, Hunter R, et al. The short-term effect of interdisciplinary medication review on function and cost in ambulatory elderly people. J Am Geriatr Soc 2004;52:93-8.

http://www.ncbi.nlm.nih.gov/entrez/query.fcgi?cmd

$=$ Retrieve $\& \mathrm{db}=$ PubMed\&list_uids $=14687321 \& \mathrm{dopt}$

$=$ Abstract

Williams PD, Williams AR, Graff JC, et al. A communitybased intervention for siblings and parents of children with chronic illness or disability: the ISEE study. $J$ Pediatr 2003; 143:386-93.

http://www.ncbi.nlm.nih.gov/entrez/query.fcgi?cmd

$=$ Retrieve\&db $=$ PubMed\&list_uids $=14517525 \& d o p t$

$=$ Abstract 
8 of 14

Winner P, Mannix LK, Putnam DG, et al. Pain-free results with sumatriptan taken at the first sign of migraine pain: 2 randomized, double-blind, placebo-controlled studies. Mayo Clin Proc 2003;78:1214-22.

http://www.ncbi.nlm.nih.gov/entrez/query.fcgi?cmd

$=$ Retrieve $\& \mathrm{db}=$ PubMed\&list uids $=14531480 \& \mathrm{dopt}$

$=$ Abstract

Woods P, Richards D. Effectiveness of nursing interventions in people with personality disorders. J Adv Nurs 2003;44:154-72.

http://www.ncbi.nlm.nih.gov/entrez/query.fcgi?cmd

$=$ Retrieve $\& \mathrm{db}=$ PubMed\&list uids $=14521682 \& \mathrm{dopt}$

$=$ Abstract

Young W, Rewa G, Goodman SG, et al. Evaluation of a community-based inner-city disease management program for postmyocardial infarction patients: a randomized controlled trial. CMAJ 2003;169:905-10.

http://www.ncbi.nlm.nih.gov/entrez/query.fcgi?cmd

$=$ Retrieve $\& \mathrm{db}=$ PubMed\&list uids $=14581307 \& \mathrm{dopt}$

$=$ Abstract

Zajicek J, Fox P, Sanders H, et al. Cannabinoids for treatment of spasticity and other symptoms related to multiple sclerosis (CAMS study): multicentre randomised placebo-controlled trial. Lancet 2003;362:1517-26.

http://www.ncbi.nlm.nih.gov/entrez/query.fcgi?cmd

$=$ Retrieve $\& \mathrm{db}=$ PubMed\&list_uids $=$ 14615106\&dopt

$=$ Abstract

Zwart S, Rovers MM, de Melker RA, et al. Penicillin for acute sore throat in children: randomised, double blind trial. BMJ 2003;327:1324-8.

http://www.ncbi.nlm.nih.gov/entrez/query.fcgi?cmd

$=$ Retrieve $\& \mathrm{db}=$ PubMed\&list_uids $=14656841 \&$ dopt

$=$ Abstract

\section{PROGNOSIS}

Bitnun A, Allen U, Heurter H, et al. Children hospitalized with severe acute respiratory syndrome-related illness in Toronto. Pediatrics 2003;112:e261.

http://www.ncbi.nlm.nih.gov/entrez/query.fcgi?cmd

$=$ Retrieve $\& \mathrm{db}=$ PubMed\&list_uids $=14523209 \& \mathrm{dopt}$

$=$ Abstract

Golden S, Boulware LE, Berkenblit G, et al. Use of glycated hemoglobin and microalbuminuria in the monitoring of diabetes mellitus. Evidence Report/Technology Assessment Number 84. AHRQ Publication No. 04-E001. Rockville, MD: Agency for Healthcare Research and Quality, October 2003.

http://www.ahcpr.gov/clinic/glycainv.htm

Saigal S, den Ouden L, Wolke D, et al. School-age outcomes in children who were extremely low birth weight from four international population-based cohorts. Pediatrics 2003;112:943-50.

http://www.ncbi.nlm.nih.gov/entrez/query.fcgi?cmd

$=$ Retrieve $\& \mathrm{db}=$ PubMed\&list_uids $=14523190 \& \mathrm{dopt}$

$=$ Abstract

Short EJ, Klein NK, Lewis BA, et al. Cognitive and academic consequences of bronchopulmonary dysplasia and very low birth weight: 8-year-old outcomes. Pediatrics 2003;112:e359-66. http://www.ncbi.nlm.nih.gov/entrez/query.fcgi?cmd $=$ Retrieve $\& \mathrm{db}=$ PubMed\&list_uids $=14595077 \& \mathrm{dopt}$ $=$ Abstract

Strik JJ, Denollet J, Lousberg R, et al. Comparing symptoms of depression and anxiety as predictors of cardiac events and increased health care consumption after myocardial infarction. J Am Coll Cardiol 2003;42:1801-7.

http://www.ncbi.nlm.nih.gov/entrez/query.fcgi?cmd $=$ Retrieve $\& \mathrm{db}=$ PubMed\&list_uids $=14642691 \& \mathrm{dopt}$

$=$ Abstract

Vollmer B, Roth S, Baudin J, et al. Predictors of long-term outcome in very preterm infants: gestational age versus neonatal cranial ultrasound. Pediatrics 2003;112:1108-14. http://www.ncbi.nlm.nih.gov/entrez/query.fcgi?cmd $=$ Retrieve $\& \mathrm{db}=$ PubMed\&list_uids $=14595054 \& \mathrm{dopt}$ $=$ Abstract

\section{CAUSATION}

Agerbo E. Risk of suicide and spouse's psychiatric illness or suicide: nested case-control study. BMJ 2003;327:1025-6.

http://www.ncbi.nlm.nih.gov/entrez/query.fcgi?cmd $=$ Retrieve $\& \mathrm{db}=$ PubMed\&list_uids $=14593038 \& d o p t$ $=$ Abstract

Allard JP, Aghdassi E, McArthur M, et al. Nutrition risk factors for survival in the elderly living in Canadian longterm care facilities. J Am Geriatr Soc 2004;52:59-65. http://www.ncbi.nlm.nih.gov/entrez/query.fcgi?cmd $=$ Retrieve $\& \mathrm{db}=$ PubMed\&list_uids $=14687316 \& d o p t$ $=$ Abstract

Bracken MB, Triche EW, Belanger K, et al. Asthma symptoms, severity, and drug therapy: a prospective study of effects on 2205 pregnancies. Obstet Gynecol 2003;102:739-52.

http://www.ncbi.nlm.nih.gov/entrez/query.fcgi?cmd $=$ Retrieve $\& \mathrm{db}=$ PubMed\&list_uids $=14551004 \& d o p t$ $=$ Abstract

Bridge JA, Day NL, Day R, et al. Major depressive disorder in adolescents exposed to a friend's suicide. J Am Acad Child Adolesc Psychiatry 2003;42:1294-300.

http://www.ncbi.nlm.nih.gov/entrez/query.fcgi?cmd $=$ Retrieve $\& \mathrm{db}=$ PubMed\&list_uids $=$ 14566166\&dopt $=$ Abstract

Cohen DA, Farley TA, Mason K. Why is poverty unhealthy? Social and physical mediators. Soc Sci Med 2003;57:1631-41. http://www.ncbi.nlm.nih.gov/entrez/query.fcgi?cmd $=$ Retrieve $\& \mathrm{db}=$ PubMed\&list_uids $=12948572 \& \mathrm{dopt}$

$=$ Abstract

Elizabeth Jesse D, Seaver W, Wallace DC. Maternal psychosocial risks predict preterm birth in a group of women from Appalachia. Midwifery 2003;19:191-202.

http://www.ncbi.nlm.nih.gov/entrez/query.fcgi?cmd $=$ Retrieve $\& \mathrm{db}=$ PubMed\&list uids $=12946335 \& \mathrm{dopt}$

$=$ Abstract

Gill TM, Kurland BF. Prognostic effect of prior disability episodes among nondisabled community-living older persons. Am J Epidemiol 2003;158:1090-6.

http://www.ncbi.nlm.nih.gov/entrez/query.fcgi?cmd $=$ Retrieve $\& \mathrm{db}=$ PubMed\&list uids $=14630605 \& \mathrm{dopt}$ $=$ Abstract 
Mora S, Redberg RF, Cui Y, et al. Ability of exercise testing to predict cardiovascular and all-cause death in asymptomatic women: a 20-year follow-up of the Lipid Research Clinics Prevalence Study. JAMA 2003;290:1600-7.

http://www.ncbi.nlm.nih.gov/entrez/query.fcgi?cmd

$=$ Retrieve $\& \mathrm{db}=$ PubMed\&list_uids = 145061 19\&dopt

$=$ Abstract

Hjalgrim LL, Westergaard T, Rostgaard K, et al. Birth weight as a risk factor for childhood leukemia: a metaanalysis of 18 epidemiologic studies. Am J Epidemiol 2003; 158:724-35.

http://www.ncbi.nlm.nih.gov/entrez/query.fcgi?cmd

$=$ Retrieve $\& \mathrm{db}=$ PubMed\&list_uids $=14561661 \& \mathrm{dopt}$

$=$ Abstract

Hviid A, Stellfeld M, Wohlfahrt J, et al. Association between thimerosal-containing vaccine and autism. JAMA 2003;290: 1763-6.

http://www.ncbi.nlm.nih.gov/entrez/query.fcgi?cmd

$=$ Retrieve $\& \mathrm{db}=$ PubMed\&list_uids $=14519711 \& \mathrm{dopt}$

$=$ Abstract

Lovato C, Linn G, Stead LF, et al. Impact of tobacco advertising and promotion on increasing adolescent smoking behaviours. Cochrane Database Syst Rev 2003;(4):CD003439.

http://www.ncbi.nlm.nih.gov/entrez/query.fcgi?cmd

$=$ Retrieve $\& \mathrm{db}=$ PubMed\&list_uids $=14583977 \& d o p t$

$=$ Abstract

Salihu HM, Shumpert MN, Slay M, et al. Childbearing beyond maternal age 50 and fetal outcomes in the United States. Obstet Gynecol 2003;102:1006-14.

http://www.ncbi.nlm.nih.gov/entrez/query.fcgi?cmd

$=$ Retrieve $\& \mathrm{db}=$ PubMed\&list_uids $=14672478 \& \mathrm{dopt}$

$=$ Abstract

Schnohr P, Scharling H, Jensen JS. Changes in leisure-time physical activity and risk of death: an observational study of 7,000 men and women. Am J Epidemiol 2003;158:639-44. http://www.ncbi.nlm.nih.gov/entrez/query.fcgi?cmd $=$ Retrieve $\& \mathrm{db}=$ PubMed\&list_uids $=14507599 \& \mathrm{dopt}$ $=$ Abstract

Smith GC, Pell JP, Dobbie R. Caesarean section and risk of unexplained stillbirth in subsequent pregnancy. Lancet 2003;362:1779-84.

http://www.ncbi.nlm.nih.gov/entrez/query.fcgi?cmd

$=$ Retrieve $\& \mathrm{db}=$ PubMed\&list_uids $=14654315 \& \mathrm{dopt}$

$=$ Abstract

Toschke AM, Montgomery SM, Pfeiffer U, et al. Early intrauterine exposure to tobacco-inhaled products and obesity. Am J Epidemiol 2003;158:1068-74.

http://www.ncbi.nlm.nih.gov/entrez/query.fcgi?cmd

$=$ Retrieve $\& \mathrm{db}=$ PubMed\&list_uids $=14630602 \& \mathrm{dopt}$

$=$ Abstract

Welborn TA, Dhaliwal SS, Bennett SA. Waist-hip ratio is the dominant risk factor predicting cardiovascular death in Australia. Med J Aust 2003;179:580-5.

http://www.ncbi.nlm.nih.gov/entrez/query.fcgi?cmd

$=$ Retrieve $\& \mathrm{db}=$ PubMed\&list_uids $=14636121 \& \mathrm{dopt}$

$=$ Abstract

Wilson RS, Bienias JL, Mendes de Leon CF, et al. Negative affect and mortality in older persons. Am $J$ Epidemiol 2003; 158:827-35. http://www.ncbi.nlm.nih.gov/entrez/query.fcgi?cmd

$=$ Retrieve $\& \mathrm{db}=$ PubMed\&list_uids $=14585760 \&$ dopt

$=$ Abstract

\section{QUALITY IMPROVEMENT}

Fine MJ, Stone RA, Lave JR, et al. Implementation of an evidence-based guideline to reduce duration of intravenous antibiotic therapy and length of stay for patients hospitalized with community-acquired pneumonia: a randomized controlled trial. Am J Med 2003;115:343-51.

http://www.ncbi.nlm.nih.gov/entrez/query.fcgi?cmd

$=$ Retrieve $\& \mathrm{db}=$ PubMed\&list_uids $=14553868 \& \mathrm{dopt}$

$=$ Abstract

Schectman JM, Schroth WS, Verme D, et al. Randomized controlled trial of education and feedback for implementation of guidelines for acute low back pain. J Gen Intern Med 2003; 18:773-80.

http://www.ncbi.nlm.nih.gov/entrez/query.fcgi?cmd

$=$ Retrieve $\& \mathrm{db}=$ PubMed\&list_uids $=14521638 \& \mathrm{dopt}$

$=$ Abstract

\section{CLINICAL PREDICTION GUIDE}

Chunilal SD, Eikelboom JW, Attia J, et al. Does this patient have pulmonary embolism? JAMA 2003;290:2849-58.

http://www.ncbi.nlm.nih.gov/entrez/query.fcgi?cmd

$=$ Retrieve $\& \mathrm{db}=$ PubMed\&list_uids $=$ 14657070\&dopt

$=$ Abstract

Constans J, Boutinet C, Salmi LR, et al. Comparison of four clinical prediction scores for the diagnosis of lower limb deep venous thrombosis in outpatients. Am $J$ Med 2003;115:436-40.

http://www.ncbi.nlm.nih.gov/entrez/query.fcgi?cmd

$=$ Retrieve $\& \mathrm{db}=$ PubMed\&list_uids $=14563499 \& \mathrm{dopt}$

$=$ Abstract

Haydel MJ, Shembekar AD. Prediction of intracranial injury in children aged five years and older with loss of consciousness after minor head injury due to nontrivial mechanisms. Ann Emerg Med 2003;42:507-14.

http://www.ncbi.nlm.nih.gov/entrez/query.fcgi?cmd

$=$ Retrieve $\& \mathrm{db}=$ PubMed\&list_uids $=14520321 \& \mathrm{dopt}$

$=$ Abstract

Jané-Llopis E, Hosman C, Jenkins R, et al. Predictors of efficacy in depression prevention programmes. Meta-analysis. Br J Psychiatry 2003;183:384-97.

http://www.ncbi.nlm.nih.gov/entrez/query.fcgi?cmd

$=$ Retrieve $\& \mathrm{db}=$ PubMed\&list_uids $=14594912 \& \mathrm{dopt}$

$=$ Abstract

Kilgo PD, Osler TM, Meredith W. The worst injury predicts mortality outcome the best: rethinking the role of multiple injuries in trauma outcome scoring. I Trauma 2003;55:599-606.

http://www.ncbi.nlm.nih.gov/entrez/query.fcgi?cmd

$=$ Retrieve \&db $=$ PubMed\&list_uids $=14566109 \& d o p t$

$=$ Abstract

Lee DS, Austin PC, Rouleau JL, et al. Predicting mortality among patients hospitalized for heart failure: derivation and validation of a clinical model. JAMA 2003;290:2581-7.

http://www.ncbi.nlm.nih.gov/entrez/query.fcgi?cmd

$=$ Retrieve $\& \mathrm{db}=$ PubMed\&list_uids $=14625335 \& \mathrm{dopt}$

$=$ Abstract 
10 of 14

Peres Bota D, Mélot C, Lopes Ferreira F, et al. Infection Probability Score (IPS): a method to help assess the probability of infection in critically ill patients. Crit Care Med 2003;31:2579-84.

http://www.ncbi.nlm.nih.gov/entrez/query.fcgi?cmd

$=$ Retrieve $\& \mathrm{db}=$ PubMed\&list uids $=14605527 \& \mathrm{dopt}$

$=$ Abstract

Sullivan T, Haider A, DiRusso SM, et al. Prediction of mortality in pediatric trauma patients: new injury severity score outperforms Injury Severity Score in the severely injured. J Trauma 2003;55:1083-8.

http://www.ncbi.nlm.nih.gov/entrez/query.fcgi?cmd

$=$ Retrieve $\& \mathrm{db}=$ PubMed\&list_uids $=14676655 \& \mathrm{dopt}$

$=$ Abstract

\section{ECONOMICS}

Sullivan SD, Buxton M, Andersson LF, et al. Cost-effectiveness analysis of early intervention with budesonide in mild persistent asthma. J Allergy Clin Immunol 2003;112:1229-36. http://www.ncbi.nlm.nih.gov/entrez/query.fcgi?cmd $=$ Retrieve $\& \mathrm{db}=$ PubMed\&list_uids $=14657888 \& d o p t$

$=$ Abstract

Within-trial cost-effectiveness of lifestyle intervention or metformin for the primary prevention of type 2 diabetes. Diabetes Care 2003;26:2518-23.

http://www.ncbi.nlm.nih.gov/entrez/query.fcgi?cmd

$=$ Retrieve \&db $=$ PubMed\&list_uids $=12941712 \& d o p t$

$=$ Abstract

\section{QUALITATIVE}

Albaugh JA. Spirituality and life-threatening illness: a phenomenologic study. Oncol Nurs Forum 2003;30:593-8.

http://www.ncbi.nlm.nih.gov/entrez/query.fcgi?cmd

$=$ Retrieve $\& \mathrm{db}=$ PubMed\&list_uids $=12861320 \& d o p t$

$=$ Abstract

Archibald G. Patients' experiences of hip fracture. $J$ Adv Nurs 2003;44:385-92.

http://www.ncbi.nlm.nih.gov/entrez/query.fcgi?cmd

$=$ Retrieve $\& \mathrm{db}=$ PubMed\&list uids $=14651710 \& \mathrm{dopt}$

$=$ Abstract

Backett-Milburn K, Cunningham-Burley S, Davis J. Contrasting lives, contrasting views? Understandings of health inequalities from children in differing social circumstances. Soc Sci Med 2003;57:613-23.

http://www.ncbi.nlm.nih.gov/entrez/query.fcgi?cmd

$=$ Retrieve \&db = PubMed\&list_uids = 12821010\&dopt

$=$ Abstract

Battaglia TA, Finley E, Liebschutz JM. Survivors of intimate partner violence speak out: trust in the patient-provider relationship. J Gen Intern Med 2003;18:617-23.

http://www.ncbi.nlm.nih.gov/entrez/query.fcgi?cmd

$=$ Retrieve $\& \mathrm{db}=$ PubMed\&list_uids $=1291164 \&$ dopt

$=$ Abstract

Bennett S, Coggan C, Adams P. Problematising depression: young people, mental health and suicidal behaviours. Soc Sci Med 2003;57:289-99.

http://www.ncbi.nlm.nih.gov/entrez/query.fcgi?cmd

$=$ Retrieve $\& \mathrm{db}=$ PubMed\&list_uids $=12765709 \& \mathrm{dopt}$

$=$ Abstract
Bissell P, Anderson C. Supplying emergency contraception via community pharmacies in the UK: reflections on the experiences of users and providers. Soc Sci Med 2003;57:2367-78.

http://www.ncbi.nlm.nih.gov/entrez/query.fcgi?cmd

$=$ Retrieve $\& \mathrm{db}=$ PubMed\&list_uids $=14572843 \& \mathrm{dopt}$

$=$ Abstract

Bourke L. Toward understanding youth suicide in an Australian rural community. Soc Sci Med 2003;57:2355-65.

http://www.ncbi.nlm.nih.gov/entrez/query.fcgi?cmd

$=$ Retrieve $\& \mathrm{db}=$ PubMed\&list_uids $=14572842 \& \mathrm{dopt}$

$=$ Abstract

Buchman TG, Ray SE, Wax ML, et al. Families' perceptions of surgical intensive care. J Am Coll Surg 2003;196:977-83.

http://www.ncbi.nlm.nih.gov/entrez/query.fcgi?cmd

$=$ Retrieve $\& \mathrm{db}=$ PubMed\&list_uids $=12788436 \& \mathrm{dopt}$

$=$ Abstract

Caron CD, Bowers BJ. Deciding whether to continue, share, or relinquish caregiving: caregiver views. Qual Health Res 2003; 13:1252-71.

http://www.ncbi.nlm.nih.gov/entrez/query.fcgi?cmd $=$ Retrieve $\& \mathrm{db}=$ PubMed\&list_uids $=14606412 \& \mathrm{dopt}$

$=$ Abstract

Carr JM. Poetic expressions of vigilance. Qual Health Res 2003;13:1324-31.

http://www.ncbi.nlm.nih.gov/entrez/query.fcgi?cmd

$=$ Retrieve $\& \mathrm{db}=$ PubMed\&list_uids $=$ 14606416\&dopt

$=$ Abstract

Clark AM. "It's like an explosion in your life...": lay perspectives on stress and myocardial infarction. J Clin Nurs 2003;12:544-53.

http://www.ncbi.nlm.nih.gov/entrez/query.fcgi?cmd

$=$ Retrieve $\& \mathrm{db}=$ PubMed\&list_uids $=12790868 \& \mathrm{dopt}$

$=$ Abstract

Clarke JN, James S. The radicalized self: the impact on the self of the contested nature of the diagnosis of chronic fatigue syndrome. Soc Sci Med 2003;57:1387-95.

http://www.ncbi.nlm.nih.gov/entrez/query.fcgi?cmd

$=$ Retrieve \&db $=$ PubMed\&list_uids $=12927469 \& d o p t$

$=$ Abstract

Collins K, O'Cathain A. The continuum of patient satisfaction - from satisfied to very satisfied. Soc Sci Med 2003;57:2465-70.

http://www.ncbi.nlm.nih.gov/entrez/query.fcgi?cmd

$=$ Retrieve $\& \mathrm{db}=$ PubMed\&list uids $=14572851 \& \mathrm{dopt}$

$=$ Abstract

Cutchin MP. The process of mediated aging-in-place: a theoretically and empirically based model. Soc Sci Med 2003; 57:1077-90.

http://www.ncbi.nlm.nih.gov/entrez/query.fcgi?cmd

$=$ Retrieve $\& \mathrm{db}=$ PubMed\&list_uids $=12878107 \& \mathrm{dopt}$

$=$ Abstract

Davis S, Kristjanson LJ, Blight J. Communicating with families of patients in an acute hospital with advanced cancer. Cancer Nurs 2003;26:337-45.

http://www.ncbi.nlm.nih.gov/entrez/query.fcgi?cmd $=$ Retrieve $\& \mathrm{db}=$ PubMed\&list_uids $=14710794 \& \mathrm{dopt}$

$=$ Abstract 
11 of 14

Durack-Bown I, Giral P, d'Ivernois JF, et al. Patients' and physicians' perceptions and experience of hypercholesterolaemia: a qualitative study. Br J Gen Pract 2003;53:851-7. http://www.ncbi.nlm.nih.gov/entrez/query.fcgi?cmd $=$ Retrieve $\& \mathrm{db}=$ PubMed\&list_uids $=14702904 \&$ dopt $=$ Abstract

Elander J, Lusher J, Bevan D, et al. Pain management and symptoms of substance dependence among patients with sickle cell disease. Soc Sci Med 2003;57:1683-96.

http://www.ncbi.nlm.nih.gov/entrez/query.fcgi?cmd

$=$ Retrieve $\& \mathrm{db}=$ PubMed\&list_uids = 12948577\&dopt

$=$ Abstract

Espezel HJ, Canam CJ. Parent-nurse interactions: care of hospitalized children. $J$ Adv Nurs 2003;44:34-41. http://www.ncbi.nlm.nih.gov/entrez/query.fcgi?cmd

$=$ Retrieve $\& \mathrm{db}=$ PubMed\&list_uids $=12956667 \&$ dopt

$=$ Abstract

Farran CJ, Loukissa D, Perraud S, et al. Alzheimer's disease caregiving information and skills. Part I: care recipient issues and concerns. Res Nurs Health 2003;26:366-75.

http://www.ncbi.nlm.nih.gov/entrez/query.fcgi?cmd

$=$ Retrieve \&db $=$ PubMed\&list_uids = 14579257\&dopt

$=$ Abstract

Fenaughty AM, Namyniuk L. "Here's what I'd do...": condom promotion strategies proposed by high-risk women in Anchorage, Alaska. Qual Health Res 2004;14:23-38.

http://www.ncbi.nlm.nih.gov/entrez/query.fcgi?cmd

$=$ Retrieve $\& \mathrm{db}=$ PubMed\&list_uids $=14725174 \& \mathrm{dopt}$

$=$ Abstract

Fredriksson GE, Högberg U, Lundman BM. Postpartum care should provide alternatives to meet parents' need for safety, active participation, and 'bonding'. Midwifery 2003; 19:267-76.

http://www.ncbi.nlm.nih.gov/entrez/query.fcgi?cmd

$=$ Retrieve $\& \mathrm{db}=$ PubMed\&list_uids $=$ 14623506\&dopt

$=$ Abstract

Fuller J. Intercultural health care as reflective negotiated practice. West J Nurs Res 2003;25:781-97.

http://www.ncbi.nlm.nih.gov/entrez/query.fcgi?cmd

$=$ Retrieve $\& \mathrm{db}=$ PubMed\&list_uids $=$ 14596179\&dopt

$=$ Abstract

Gånemo A, Lindholm C, Lindberg M, et al. Quality of life in adults with congenital ichthyosis. J Adv Nurs 2003;44: 412-9.

http://www.ncbi.nlm.nih.gov/entrez/query.fcgi?cmd

$=$ Retrieve $\& \mathrm{db}=$ PubMed\&list_uids $=14651713 \& \mathrm{dopt}$

$=$ Abstract

Giarelli E. Bringing threat to the fore: participating in lifelong surveillance for genetic risk of cancer. Oncol Nurs Forum 2003;30:945-55.

http://www.ncbi.nlm.nih.gov/entrez/query.fcgi?cmd

$=$ Retrieve $\& \mathrm{db}=$ PubMed\&list_uids = 14603352\&dopt

$=$ Abstract

Glenton C. Chronic back pain sufferers-striving for the sick role. Soc Sci Med 2003;57:2243-52.

http://www.ncbi.nlm.nih.gov/entrez/query.fcgi?cmd

$=$ Retrieve $\& \mathrm{db}=$ PubMed\&list_uids $=14512253 \& \mathrm{dopt}$

$=$ Abstract
Gross MM, Haunschild T, Stoexen T, et al. Women's recognition of the spontaneous onset of labor. Birth 2003;30:267-71.

Holm KE, Patterson JM, Gurney JG. Parental involvement and family-centred care in the diagnostic and treatment phases of childhood cancer: results from a qualitative study. J Pediatr Onc Nurs 2003;20:301-13.

http://www.ncbi.nlm.nih.gov/entrez/query.fcgi?cmd $=$ Retrieve $\& \mathrm{db}=$ PubMed\&list_uids $=$ 14738162\&dopt

$=$ Abstract

Howteerakul N, Higginbotham N, Freeman S, et al. ORS is never enough: physician rationales for altering standard treatment guidelines when managing childhood diarrhoea in Thailand. Soc Sci Med 2003;57:1031-44.

http://www.ncbi.nlm.nih.gov/entrez/query.fcgi?cmd

$=$ Retrieve\&db $=$ PubMed\&list_uids = 12878103\&dopt

$=$ Abstract

Hsu HC, Shyu YI. Implicit exchanges in family caregiving for frail elders in Taiwan. Qual Health Res 2003;13:1078-93.

http://www.ncbi.nlm.nih.gov/entrez/query.fcgi?cmd

$=$ Retrieve \&db $=$ PubMed\&list_uids $=14556420 \& d o p t$

$=$ Abstract

Ingram J, Johnson D, Hamid N. South Asian grandmothers' influence on breast feeding in Bristol. Midwifery 2003; 19:318-27.

http://www.ncbi.nlm.nih.gov/entrez/query.fcgi?cmd

$=$ Retrieve \&db $=$ PubMed\&list_uids = 14623511\&dopt

$=$ Abstract

Johnson JL, Lovato CY, Maggi S, et al. Smoking and adolescence: narratives of identity. Res Nurs Health 2003;26:387-97.

http://www.ncbi.nlm.nih.gov/entrez/query.fcgi?cmd

$=$ Retrieve\&db $=$ PubMed\&list_uids $=14579259 \&$ dopt

$=$ Abstract

Jones PS, Zhang XE, Meleis AI. Transforming vulnerability. West J Nurs Res 2003;25:835-53.

http://www.ncbi.nlm.nih.gov/entrez/query.fcgi?cmd

$=$ Retrieve \&db $=$ PubMed\&list_uids = 14596182\&dopt

$=$ Abstract

Kilian R, Lindenbach I, Löbig $\mathrm{U}$, et al. Indicators of empowerment and disempowerment in the subjective evaluation of the psychiatric treatment process by persons with severe and persistent mental illness: a qualitative and quantitative analysis. Soc Sci Med 2003;57:1127-42.

http://www.ncbi.nlm.nih.gov/entrez/query.fcgi?cmd

$=$ Retrieve $\& \mathrm{db}=$ PubMed\&list_uids = 12878111\&dopt

$=$ Abstract

Kirkman M. Parents' contributions to the narrative identity of offspring of donor-assisted conception. Soc Sci Med 2003; 57:2229-42.

http://www.ncbi.nlm.nih.gov/entrez/query.fcgi?cmd

$=$ Retrieve \&db $=$ PubMed\&list_uids = 14512252\&dopt

$=$ Abstract

Kirkham SR. The politics of belonging and intercultural health care. West J Nurs Res 2003;25:762-80.

http://www.ncbi.nlm.nih.gov/entrez/query.fcgi?cmd

$=$ Retrieve $\& \mathrm{db}=$ PubMed\&list_uids $=$ 14596178\&dopt

$=$ Abstract 
12 of 14

Lepp M, Ringsberg KC, Holm AK, et al. Dementia-involving patients and their caregivers in a drama programme: the caregivers' experiences. J Clin Nurs 2003;12:873-81. http://www.ncbi.nlm.nih.gov/entrez/query.fcgi?cmd $=$ Retrieve $\& \mathrm{db}=$ PubMed\&list_uids $=14632980 \& d o p t$

$=$ Abstract

Lester H, Tritter JQ, England E. Satisfaction with primary care: the perspectives of people with schizophrenia. Fam Pract 2003;20:508-13.

http://www.ncbi.nlm.nih.gov/entrez/query.fcgi?cmd

$=$ Retrieve $\& \mathrm{db}=$ PubMed\&list_uids $=14507789 \& \mathrm{dopt}$

$=$ Abstract

Lichtenstein B. Stigma as a barrier to treatment of sexually transmitted infection in the American deep south: issues of race, gender and poverty. Soc Sci Med 2003;57:2435-45.

http:/www.ncbi.nlm.nih.gov/entrez/query.fcgi?cmd

$=$ Retrieve $\& \mathrm{db}=$ PubMed\&list_uids $=14572849 \& \mathrm{dopt}$

$=$ Abstract

Lillrank A. Back pain and the resolution of diagnostic uncertainty in illness narratives. Soc Sci Med 2003;57:1045-54. http://www.ncbi.nlm.nih.gov/entrez/query.fcgi?cmd $=$ Retrieve $\& \mathrm{db}=$ PubMed\&list_uids $=12878104 \& \mathrm{dopt}$

$=$ Abstract

Lipson JG, Weinstein HM, Gladstone EA, et al. Bosnian and Soviet refugees' experiences with health care. West $J$ Nurs Res 2003;25:854-71.

http://www.ncbi.nlm.nih.gov/entrez/query.fcgi?cmd

$=$ Retrieve $\& \mathrm{db}=$ PubMed\&list_uids $=14596183 \& d o p t$

$=$ Abstract

Lowton K. Only when I cough? Adults' disclosure of cystic fibrosis. Qual Health Res 2004;14:167-86.

Madi BC, Crow R. A qualitative study of information about available options for childbirth venue and pregnant women's preference for a place of delivery. Midwifery 2003;19:328-36. http://www.ncbi.nlm.nih.gov/entrez/query.fcgi?cmd $=$ Retrieve $\& \mathrm{db}=$ PubMed\&list_uids $=14623512 \& d o p t$

$=$ Abstract

Meade CD, Calvo A, Rivera MA, et al. Focus groups in the design of prostate cancer screening information for Hispanic farmworkers and African American men. Oncol Nurs Forum 2003;30:967-75.

http://www.ncbi.nlm.nih.gov/entrez/query.fcgi?cmd

$=$ Retrieve\&db $=$ PubMed\&list_uids = 14603354\&dopt

$=$ Abstract

Meeker MA. Family surrogate decision making at the end of life: seeing them through with care and respect. Qual Health Res 2004; 14:204-25.

Mendelson C. Gentle hugs: Internet listservs as sources of support for women with lupus. ANS Adv Nurs Sci 2003;26:299306.

http://www.ncbi.nlm.nih.gov/entrez/query.fcgi?cmd

$=$ Retrieve $\& \mathrm{db}=$ PubMed\&list_uids $=14674578 \& d o p t$

$=$ Abstract

Mielke J, Martin DK, Singer PA. Priority setting in a hospital critical care unit: qualitative case study. Crit Care Med 2003;31:2764-8.

http://www.ncbi.nlm.nih.gov/entrez/query.fcgi?cmd

$=$ Retrieve $\& \mathrm{db}=$ PubMed\&list_uids $=14668612 \& d o p t$

$=$ Abstract
Mohr WK. Surfacing the life phases of a mental health support group. Qual Health Res 2004;14:61-77.

http://www.ncbi.nlm.nih.gov/entrez/query.fcgi?cmd

$=$ Retrieve $\& \mathrm{db}=$ PubMed\&list_uids $=$ 14725176\&dopt

$=$ Abstract

Morse JM, Beres MA, Spiers JA, et al. Identifying signals of suffering by linking verbal and facial cues. Qual Health Res 2003;13:1063-77.

http://www.ncbi.nlm.nih.gov/entrez/query.fcgi?cmd

$=$ Retrieve $\& \mathrm{db}=$ PubMed\&list_uids $=14556419 \& d o p t$

$=$ Abstract

Murphy DJ, Pope C, Frost J, et al. Women's views on the impact of operative delivery in the second stage of labour: qualitative interview study. $B M J$ 2003;327:1132-6.

http://www.ncbi.nlm.nih.gov/entrez/query.fcgi?cmd $=$ Retrieve $\& \mathrm{db}=$ PubMed\&list_uids $=14615336 \& d o p t$

$=$ Abstract

Nicolson P, Burr J. What is 'normal' about women's (hetero)sexual desire and orgasm?: a report of an in-depth interview study. Soc Sci Med 2003;57:1735-45.

http://www.ncbi.nlm.nih.gov/entrez/query.fcgi?cmd $=$ Retrieve \&db $=$ PubMed\&list_uids $=12948581 \& d o p t$

$=$ Abstract

Nixon S, Renwick R. Experiences of contemplating returning to work for people living with HIV/AIDS. Qual Health Res 2003; 13:1272-90.

http://www.ncbi.nlm.nih.gov/entrez/query.fcgi?cmd $=$ Retrieve $\& \mathrm{db}=$ PubMed\&list_uids $=14606413 \& d o p t$

$=$ Abstract

O'Baugh J, Wilkes LM, Luke S, et al. 'Being positive': perceptions of patients with cancer and their nurses. $J A d v$ Nurs 2003;44:262-70.

http://www.ncbi.nlm.nih.gov/entrez/query.fcgi?cmd

$=$ Retrieve $\& \mathrm{db}=$ PubMed\&list_uids $=$ 14641396\&dopt

$=$ Abstract

Owen D, Vidal-Alaball J, Mansour M, et al. Parent's opinions on the diagnosis of children under 2 years of age with urinary tract infection. Fam Pract 2003;20:531-7.

http://www.ncbi.nlm.nih.gov/entrez/query.fcgi?cmd $=$ Retrieve $\& \mathrm{db}=$ PubMed\&list uids $=14507794 \& d o p t$

$=$ Abstract

Pfeffer N. Screening for breast cancer: candidacy and compliance. Soc Sci Med 2004;58:151-60.

http://www.ncbi.nlm.nih.gov/entrez/query.fcgi?cmd

$=$ Retrieve \&db = PubMed\&list_uids = 14572928\&dopt

$=$ Abstract

Phinney A, Wallhagen M. Recognizing and understanding the symptoms of type 2 diabetes. Can J Nurs Res 2003;35:108-24

http://www.ncbi.nlm.nih.gov/entrez/query.fcgi?cmd

$=$ Retrieve $\& \mathrm{db}=$ PubMed\&list_uids $=14746124 \& d o p t$

$=$ Abstract

Pinto KC. Intersections of gender and age in health care: adapting autonomy and confidentiality for the adolescent girl. Qual Health Res 2004;14:78-99.

http://www.ncbi.nlm.nih.gov/entrez/query.fcgi?cmd

$=$ Retrieve $\& \mathrm{db}=$ PubMed\&list_uids $=14725177 \& \mathrm{dopt}$

$=$ Abstract 
Porter EJ. Moments of apprehension in the midst of a certainty: some frail older widows' lives with a personal emergency response system. Qual Health Res 2003;13: $1311-23$.

http://www.ncbi.nlm.nih.gov/entrez/query.fcgi?cmd

$=$ Retrieve $\& \mathrm{db}=$ PubMed\&list uids $=14606413 \& \mathrm{dopt}$

$=$ Abstract

Rodrigues M, Patel V, Jaswal S, et al. Listening to mothers: qualitative studies on motherhood and depression from Goa, India. Soc Sci Med 2003;57:1797-806.

http://www.ncbi.nlm.nih.gov/entrez/query.fcgi?cmd

$=$ Retrieve $\& \mathrm{db}=$ PubMed\&list uids $=14499506 \& \mathrm{dopt}$

$=$ Abstract

Schmidt LA. Patients' perceptions of nursing care in the hospital setting. $J$ Adv Nurs 2003;44:393-9.

http://www.ncbi.nlm.nih.gov/entrez/query.fcgi?cmd

$=$ Retrieve $\& \mathrm{db}=$ PubMed\&list_uids $=14651711 \& \mathrm{dopt}$

$=$ Abstract

Schwarz JK. Understanding and responding to patients' requests for assistance in dying. J Nurs Scholarsh 2003;35:37784.

http://www.ncbi.nlm.nih.gov/entrez/query.fcgi?cmd

$=$ Retrieve $\& \mathrm{db}=$ PubMed\&list_uids $=14735682 \& \mathrm{dopt}$

$=$ Abstract

Sinding C. Disarmed complaints: unpacking satisfaction with end-of-life care. Soc Sci Med 2003;57:1375-85.

http://www.ncbi.nlm.nih.gov/entrez/query.fcgi?cmd

$=$ Retrieve $\& \mathrm{db}=$ PubMed\&list uids $=12927468 \& \mathrm{dopt}$

$=$ Abstract

Skovdahl K, Kihlgren AL, Kihlgren M. Dementia and aggressiveness: video recorded morning care from different care units. J Clin Nurs 2003;12:888-98.

http://www.ncbi.nlm.nih.gov/entrez/query.fcgi?cmd

$=$ Retrieve $\& \mathrm{db}=$ PubMed\&list uids $=14632982 \& \mathrm{dopt}$

$=$ Abstract

Smith SM, O'Leary M, Bury G, et al. A qualitative investigation of the views and health beliefs of patients with Type 2 diabetes following the introduction of a diabetes shared care service. Diabet Med 2003;20:853-7.

http://www.ncbi.nlm.nih.gov/entrez/query.fcgi?cmd

$=$ Retrieve $\& \mathrm{db}=$ PubMed\&list uids $=14510868 \& \mathrm{dopt}$

$=$ Abstract

Struthers R, Hodge FS, Geishirt-Cantrell B, et al. Participant experiences of Talking Circles on type 2 diabetes in two Northern Plains American Indian Tribes. Qual Health Res 2003:13:1094-115.

http://www.ncbi.nlm.nih.gov/entrez/query.fcgi?cmd

$=$ Retrieve $\& \mathrm{db}=$ PubMed\&list_uids $=14556421 \& \mathrm{dopt}$

$=$ Abstract

Taylor SD. Predictive genetic test decisions for Huntington's disease: context, appraisal and new moral imperatives. Soc Sci Med 2004;58:137-49.

http://www.ncbi.nlm.nih.gov/entrez/query.fcgi?cmd

$=$ Retrieve $\& \mathrm{db}=$ PubMed\&list_uids $=14572927 \& d o p t$

$=$ Abstract

Thompson T, Barbour R, Schwartz L. Adherence to advance directives in critical care decision making: vignette study. BMJ 2003·327:1011-7. http://www.ncbi.nlm.nih.gov/entrez/query.fcgi?cmd

$=$ Retrieve $\& \mathrm{db}=$ PubMed\&list_uids $=14593032 \& \mathrm{dopt}$

$=$ Abstract

Thulesius H, Håkansson A, Petersson K. Balancing: a basic process in end-of-life cancer care. Qual Health Res 2003; 13:1353-77.

http://www.ncbi.nlm.nih.gov/entrez/query.fcgi?cmd $=$ Retrieve $\& \mathrm{db}=$ PubMed\&list_uids $=14658351 \& \mathrm{dopt}$

$=$ Abstract

Tierney CD, Yusuf H, McMahon SR, et al. Adoption of reminder and recall messages for immunizations by pediatricians and public health clinics. Pediatrics 2003; 112:1076-82

http://www.ncbi.nlm.nih.gov/entrez/query.fcgi?cmd

$=$ Retrieve \&db $=$ PubMed\&list_uids $=14595049 \&$ dopt

$=$ Abstract

Van Til L, MacQuarrie C, Herbert R. Understanding the barriers to cervical cancer screening among older women. Qual Health Res 2003;13:1116-31.

http://www.ncbi.nlm.nih.gov/entrez/query.fcgi?cmd $=$ Retrieve $\& \mathrm{db}=$ PubMed\&list_uids $=14556422 \& \mathrm{dopt}$

$=$ Abstract

Vig EK, Pearlman RA. Quality of life while dying: a qualitative study of terminally ill older men. $J$ Am Geriatr Soc 2003;51:1595-601.

http://www.ncbi.nlm.nih.gov/entrez/query.fcgi?cmd

$=$ Retrieve $\& \mathrm{db}=$ PubMed\&list uids $=14687389 \& \mathrm{dopt}$

$=$ Abstract

Volker DL. Assisted dying and end-of-life symptom management. Cancer Nurs 2003;26:392-9.

http://www.ncbi.nlm.nih.gov/entrez/query.fcgi?cmd

$=$ Retrieve $\& \mathrm{db}=$ PubMed\&list_uids $=14710801 \& \mathrm{dopt}$

$=$ Abstract

Vuckovic N, Harris EL, Valanis B, et al. Consumer knowledge and opinions of genetic testing for breast cancer risk. Am J Obstet Gynecol 2003;189:S48-53.

http://www.ncbi.nlm.nih.gov/entrez/query.fcgi?cmd

$=$ Retrieve $\& \mathrm{db}=$ PubMed\&list_uids $=14586321 \& \mathrm{dopt}$

$=$ Abstract

Ward-Griffin C, Bol N, Kim H, et al. Relationships between families and registered nurses in long-term-care facilities: a critical analysis. Can J Nurs Res 2003;35:150-74. http://www.ncbi.nlm.nih.gov/entrez/query.fcgi?cmd

$=$ Retrieve $\& \mathrm{db}=$ PubMed\&list_uids $=14746126 \& d o p t$

$=$ Abstract

Weaver T, Tyrer P, Ritchie J, et al. Assessing the value of assertive outreach. Qualitative study of process and outcome generation in the UK700 trial. Br J Psychiatry 2003;183:43745 .

http://www.ncbi.nlm.nih.gov/entrez/query.fcgi?cmd

$=$ Retrieve \&db $=$ PubMed\&list_uids $=14594920 \& d o p t$

$=$ Abstract

Wilde MH. Life with an indwelling urinary catheter: the dialectic of stigma and acceptance. Qual Health Res 2003;13:1189-204

http://www.ncbi.nlm.nih.gov/entrez/query.fcgi?cmd $=$ Retrieve $\& \mathrm{db}=$ PubMed\&list_uids $=14606409 \& \mathrm{dopt}$

$=$ Abstract 
14 of 14

Wilton R. Putting policy into practice? Poverty and people with serious mental illness. Soc Sci Med 2004;58:25-39.

http://www.ncbi.nlm.nih.gov/entrez/query.fcgi?cmd

$=$ Retrieve $\& \mathrm{db}=$ PubMed\&list_uids $=14572919 \& \mathrm{dopt}$

$=$ Abstract

Wind S, Van Sickle D, Wright AL. Health, place and childhood asthma in southwest Alaska. Soc Sci Med 2004;58:75-88. http://www.ncbi.nlm.nih.gov/entrez/query.fcgi?cmd

$=$ Retrieve $\& \mathrm{db}=$ PubMed\&list_uids $=14572922 \& \mathrm{dopt}$

$=$ Abstract

Wong MKY, Crawford TJ, Gask L, et al. A qualitative investigation into women's experiences after a miscarriage: implications for the primary healthcare team. Br J Gen Pract 2003;53:697-702.

Yeh CH. Dynamic coping behaviors and process of parental response to child's cancer. Appl Nurs Res 2003;16:245-55. http://www.ncbi.nlm.nih.gov/entrez/query.fcgi?cmd

$=$ Retrieve $\& \mathrm{db}=$ PubMed\&list_uids $=14608558 \& \mathrm{dopt}$

$=$ Abstract

Young AJ, Ofori-Boateng T, Rodriguez KL, et al. Meaning and agency in discussing end-of-life care: a study of elderly veterans' values and interpretations. Qual Health Res 2003;13:1039-62.

http://www.ncbi.nlm.nih.gov/entrez/query.fcgi?cmd

$=$ Retrieve $\& \mathrm{db}=$ PubMed\&list_uids $=14556418 \& \mathrm{dopt}$

$=$ Abstract

Zhang AY, Siminoff LA. The role of the family in treatment decision making by patients with cancer. Oncol Nurs Forum 2003;30:1022-8.

http://www.ncbi.nlm.nih.gov/entrez/query.fcgi?cmd $=$ Retrieve \&db $=$ PubMed\&list_uids $=14603359 \&$ dopt $=$ Abstract 